\title{
Maximal lensing: mass constraints on point lens configurations
}

\author{
Johann Bayer • Charles C. Dyer
}

Published online: 10 February 2009

(C) Springer Science+Business Media, LLC 2009

\section{Erratum to: Gen Relativ Gravit (2007) 39:1413-1418 DOI 10.1007/s10714-007-0463-x}

The original version of the article unfortunately contained mistakes. There are numerical corrections in two paragraphs (Sect. 3, p. 1417)—see below.

In order to translate this result into astrophysically relevant quantities, dimensions must be recovered in the lens equation (2). As noted in the previous section, $R_{E}$, the Einstein radius of the total mass for the lenses in the polygon is used as the normalization factor for distances on the lens plane. Therefore, $R_{E}=\sqrt{D \Phi M} \equiv 1$, where $M$ is the total mass of the deflectors at the vertices of the regular polygon, $\Phi \equiv \frac{4 G}{c^{2}} \cong 6.25 \times 10^{-13} \mathrm{ly} / \mathrm{M}_{\odot}$ and the distance factor, $D=\frac{D_{O L} D_{L S}}{D_{O S}}$, depends on the distances from observer to lens, lens to source and observer to source, as indicated in the preceding section.

Looking at a situation inside the Galaxy, we set the total mass of the deflectors to be $M=3.5 \mathrm{M}_{\odot}$ and the distances to lens and source to be respectively $D_{O L}=450$ ly and $D_{O S}=1350 \mathrm{ly}$. These choices lead to an Einstein radius of $R_{E}=1.62$ au and therefore a critical radius of $r_{c r}=1.18 \mathrm{au}$. Furthermore, this implies $m_{\star}<0.07 \mathrm{M}_{\odot}$, making this lensing case sensitive to brown dwarfs and planets, but not to more massive stars.

The online version of the original article can be found under doi:10.1007/s10714-007-0463-x.

\footnotetext{
J. Bayer $(\varangle)$

Department of Physics, University of Toronto, 60 St. George St., Toronto, ON M5S 1A7, Canada e-mail: jbayer@physics.utoronto.ca

C. C. Dyer

Department of Astronomy and Astrophysics, University of Toronto, 50 St. George St., Toronto, ON M5S 3H4, Canada e-mail: dyer@astro.utoronto.ca
} 